\title{
Prise en Charge des Hernies de l'aine : Expérience d'un Hôpital Confessionnel en Zone Rurale au Togo
}

\author{
Biréga Koutora, \\ Service de Chirurgie Générale, Hôpital Saint Jean de Dieu d'Afagnan, Togo \\ Fousséni Alassani, \\ Service de Chirurgie Viscérale A, CHU Sylvanus Olympio, Lomé \\ Faculté des Sciences de la Santé, Université de Lomé, Togo
}

Efoé-Ga Amouzou,

Service de Chirurgie Générale B, CHU Sylvanus Olympio, Lomé

Faculté des Sciences de la Santé, Université de Lomé, Togo

Abossisso Sakiye,

Service des Urgences Chirurgicales, CHU Sylvanus Olympio, Lomé

Faculté des Sciences de la Santé, Université de Lomé, Togo

Raouf Fayissal Geraldo,

Service de Chirurgie Générale B, CHU Sylvanus Olympio, Lomé

Faculté des Sciences de la Santé, Université de Lomé, Togo

Boyodi Katanga Tchangai,

Service de Chirurgie Viscérale A, CHU Sylvanus Olympio, Lomé

Faculté des Sciences de la Santé, Université de Lomé, Togo

Kokou Kouliwa Kanassoua,

Service des Urgences Chirurgicales, CHU Sylvanus Olympio, Lomé

Faculté des Sciences de la Santé, Université de Lomé, Togo

Boniface Apetogbo,

Simona Villa,

Service de Chirurgie Générale, Hôpital Saint Jean de Dieu d'Afagnan, Togo

Badjona Songne-Gnamkoulamba,

Service des Urgences Chirurgicales, CHU Sylvanus Olympio, Lomé

Faculté des Sciences de la Santé, Université de Lomé, Togo

Doi: 10.19044/esj.2019.v15n18p191 URL:http://dx.doi.org/10.19044/esj.2019.v15n18p191

\section{Résumé}

Objectif : Décrire la prise en charge des hernies de l'aine en zone rurale au Togo. Méthodologie : Etude transversale descriptive, incluant les patients pris en charge pour hernies de l'aine à l'Hôpital Saint Jean de Dieu d'Afagnan, de Janvier à Septembre 2018 (9 mois). Résultats : Cent soixanteun patients ont été opérés de hernies de l'aine. Il y avait 146 hommes et 15 
femmes, avec une moyenne d'âge de 40,52 $\pm 20,02$ ans (extrêmes : 1 an et 80 ans). Il s'agissait essentiellement des personnes exerçant des activités de force. $62,73 \%$ des patients avaient une hernie inguino-scrotale, 32,30\% avaient une hernie inguinale et $4,97 \%$ une hernie crurale. La hernie siégeait à droite dans $54,66 \%$ des cas, à gauche dans $36,02 \%$ des cas et dans $9,32 \%$ des cas elle était bilatérale. La prise en charge a été faite en chirurgie programmée pour la majorité des patients. Treize patients $(8,07 \%)$ ont été opérés en urgence pour hernies étranglées. La technique de Shouldice était majoritairement utilisée pour les hernies inguinales et inguino-scrotales (147 cas) et la technique de Mac Vay pour les hernies crurales. Les suites opératoires étaient simples dans 157 cas (97,52\%), et émaillées de complications dans 4 cas $(2,48 \%)$. Conclusion : Les hernies de l'aine sont courantes en pratique chirurgicale rurale. Les techniques classiques d'herniorraphie constituent leur principal moyen thérapeutique.

Mots-clés : Hernies de l'aine, Traitement, Zone rurale 


\title{
Care for Groin Hernias: Experience of a Denominational Hospital in Rural Zone in Togo
}

\author{
Biréga Koutora, \\ Service de Chirurgie Générale, Hôpital Saint Jean de Dieu d'Afagnan, Togo \\ Fousséni Alassani, \\ Service de Chirurgie Viscérale A, CHU Sylvanus Olympio, Lomé \\ Faculté des Sciences de la Santé, Université de Lomé, Togo
}

Efoé-Ga Amouzou,

Service de Chirurgie Générale B, CHU Sylvanus Olympio, Lomé

Faculté des Sciences de la Santé, Université de Lomé, Togo

Abossisso Sakiye,

Service des Urgences Chirurgicales, CHU Sylvanus Olympio, Lomé

Faculté des Sciences de la Santé, Université de Lomé, Togo

Raouf Fayissal Geraldo,

Service de Chirurgie Générale B, CHU Sylvanus Olympio, Lomé

Faculté des Sciences de la Santé, Université de Lomé, Togo

Boyodi Katanga Tchangai,

Service de Chirurgie Viscérale A, CHU Sylvanus Olympio, Lomé

Faculté des Sciences de la Santé, Université de Lomé, Togo

Kokou Kouliwa Kanassoua,

Service des Urgences Chirurgicales, CHU Sylvanus Olympio, Lomé

Faculté des Sciences de la Santé, Université de Lomé, Togo

Boniface Apetogbo,

Simona Villa,

Service de Chirurgie Générale, Hôpital Saint Jean de Dieu d'Afagnan, Togo

Badjona Songne-Gnamkoulamba,

Service des Urgences Chirurgicales, CHU Sylvanus Olympio, Lomé

Faculté des Sciences de la Santé, Université de Lomé, Togo

\begin{abstract}
Background: This paper focuses on describing the management of groin hernias in rural zone in Togo. Methodology: It is a descriptive transversal study, which include patients treated for groin hernias, at Saint Jean de Dieu Hospital of Afagnan, from January to September 2018 (9 months). Results: One hundred and sixty-one (161) patients were operated for groin hernias. They comprised of 146 men and 15 women, with an average
\end{abstract}


age of $40.52 \pm 20.02$ years old (extremes: 1 year and 80 years old). They were mainly people involved in strenuous activities. $62.73 \%$ of the patients had inguinoscrotal hernia, 32.30\% had inguinal hernia, and $4.97 \%$ had femoral hernia. The hernia was located on the right in $54.66 \%$ of cases, and on the left in $36.02 \%$ of cases. It was bilateral in $9.32 \%$ of cases. The majority of patients were operated during operating program. 13 patients $(8.07 \%)$ underwent an emergency operation for strangulated hernias. Shouldice technique was used for inguinal and inguinoscrotal hernias (147 cases), and Mac Vay technique was used for femoral hernias. The postoperative course was simple in 157 cases $(97.52 \%)$ and complicated in 4 cases $(2.48 \%)$. Conclusion: Groin hernias are common pathologies in rural surgical practice. Classical herniorraphy techniques are used majorly for its treatment.

Keywords: Groin hernias, Treatment, Rural zone

\section{Introduction}

Les hernies de l'aine sont les hernies de la région inguino-fémorale, région frontière entre l'abdomen et la cuisse. Elles comprennent les hernies inguinales et les hernies fémorales encore appelées hernies crurales. En dehors des hernies congénitales qui sont dues à la persistance du canal péritonéo-vaginal, elles ont toutes une cause commune qui est l'affaiblissement du fascia transversalis au niveau de l'orifice musculopectinéal de Fruchaud (Wind, 2002). Ce sont des affections fréquentes, surtout chez l'homme, atteignant aussi bien l'enfant que l'adulte (Wind, 2002 ; Mukuku, 2014). Le diagnostic est basé sur l'examen clinique. Le traitement de ces affections est chirurgical et il existe plusieurs procédés (Fingerhut, 2008). Le choix d'un procédé thérapeutique dépend de plusieurs facteurs dont les réalités locales. Si dans les pays développés la prise en charge des hernies de l'aine a connu une révolution avec l'avènement des plaques prothétiques et de la cœlioscopie, dans les pays en développement, et surtout dans les hôpitaux périphériques, les procédés classiques d'herniorraphie constituent souvent les seuls moyens thérapeutiques à proposer aux patients. Cette étude a été initiée afin de décrire les modalités de prise en charge des hernies de l'aine dans un hôpital confessionnel situé en zone rurale au Togo.

\section{Méthodologie}

Il s'est agi d'une étude transversale descriptive menée à l'Hôpital Saint Jean de Dieu d'Afagnan de Janvier à Septembre 2018 (9 mois). Etaient inclus dans l'étude, les patients pris en charge pour hernies de l'aine. Les signes fonctionnels étaient : la tuméfaction, la douleur, et les tiraillements de l'aine. Le principal signe physique était la tuméfaction siégeant à l'aine. Les 
caractères douloureux, réductible, impulsif et expansif de la tuméfaction étaient précisés de même que ses dimensions et le siège du collet. Les patients pris en charge pour des hernies d'autres points faibles de la paroi abdominale ont été exclus de l'étude.

Dans la démarche de la prise en charge, le patient était admis et opéré en urgence après réalisation d'un bilan préopératoire d'urgence lorsque la hernie était étranglée ; puis hospitalisé dans le service de pédiatrie si le patient est âgé de moins de 15 ans, ou dans le service de chirurgie générale si le patient a plus de 15 ans. Lorsque la hernie n'était pas étranglée, le patient était admis en consultation régulière, il réalisait un bilan préopératoire complet, et était programmé pour l'intervention chirurgicale. Il rentrait à l'hôpital la veille de son jour d'opération pour être hospitalisé soit dans le service de pédiatrie s'il a moins de 15 ans, soit dans le service de chirurgie générale s'il a plus de 15 ans. Le contrôle postopératoire était systématique à deux semaines post-opératoires, puis à un mois et trois mois du premier contrôle.

Les paramètres étudiés étaient : les paramètres épidémiologiques (le nombre de patients, l'âge, le sexe, la profession), les aspects cliniques (circonstances de découverte, délai d'évolution, et examen physique), les aspects thérapeutiques (contexte d'urgence ou non, technique opératoire, gestes associés) et évolutifs (à court, moyen et long terme).

Le traitement des données a été fait à partir du tableur Excel 2013. Les images ont été traitées sur logiciel Paint.

\section{Résultats}

\section{Aspects épidémiologiques}

Durant la période d'étude, 831 patients ont été opérés dans le service dont 209 (25,15\%) de hernies de la paroi abdominale. Parmi les 209 patients, $161(77,03 \%)$ étaient opérés de hernies de l'aine. Cent soixante-seize hernies de l'aine ont été diagnostiquées et traitées chez les 161 patients. Il y avait 146 hommes $(90,68 \%)$ et 15 femmes $(9,32 \%)$, soit un sex-ratio de 9,73. L'âge moyen était de 40,52 $\pm 20,02$ ans (extrêmes : 1 an et 80 ans). La tranche d'âge de 45 à 60 ans était la plus représentée (48 cas / 29,81\%). Selon la profession, $16,77 \%$ des patients étaient des agriculteurs, $13,66 \%$ étaient mécaniciens, $13,04 \%$ revendeurs, $11,80 \%$ menuisiers, et 9,94\% maçons. Les paramètres épidémiologiques sont récapitulés dans le Tableau I.

\section{Aspects cliniques}

Les circonstances de découverte étaient la tuméfaction de l'aine (161 cas / 100\%), les douleurs (13 cas / 8,07\%) et les tiraillements de l'aine (6 cas / 3,73\%). L'examen physique retrouvait une hernie de l'aine simple dans 148 cas, et une hernie étranglée dans 13 cas. Le délai d'évolution de la hernie le plus court était de 5 mois, et le plus long de 23 ans. La hernie était inguinale 
pure chez 52 patients, inguino-scrotale chez 101 patients, et crurale chez 8 patients. Les figures 1, 2 et 3 sont des exemples illustratifs des différents types de hernie de l'aine retrouvés dans notre série. La hernie siégeait à droite dans $54,66 \%$ des cas, à gauche dans $36,02 \%$ des cas, et elle était bilatérale dans $9,32 \%$ des cas.

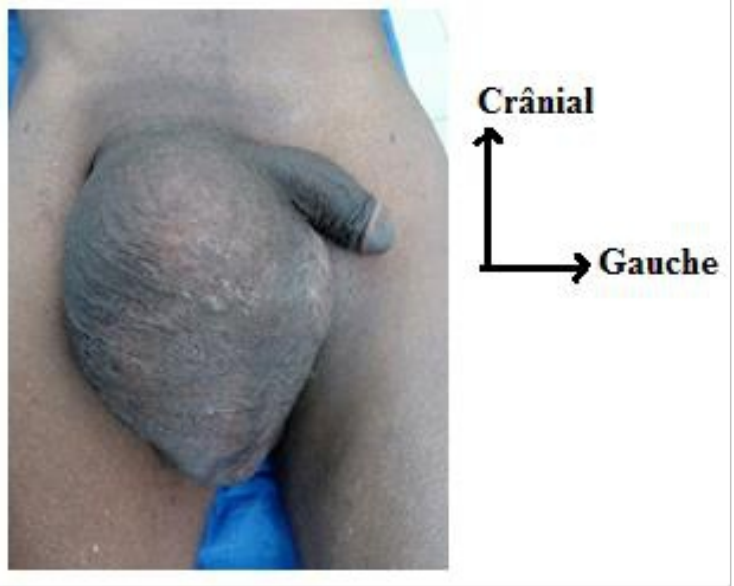

Figure 1. Hernie inguino-scrotale droite

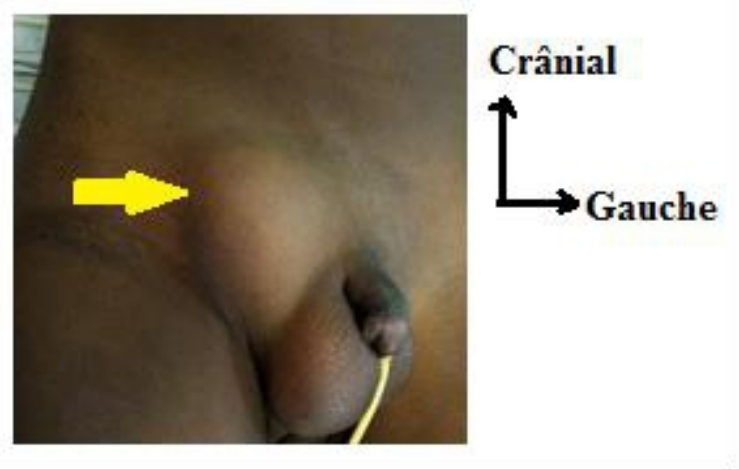

Figure 2. Hernie inguinale droite (flèche)

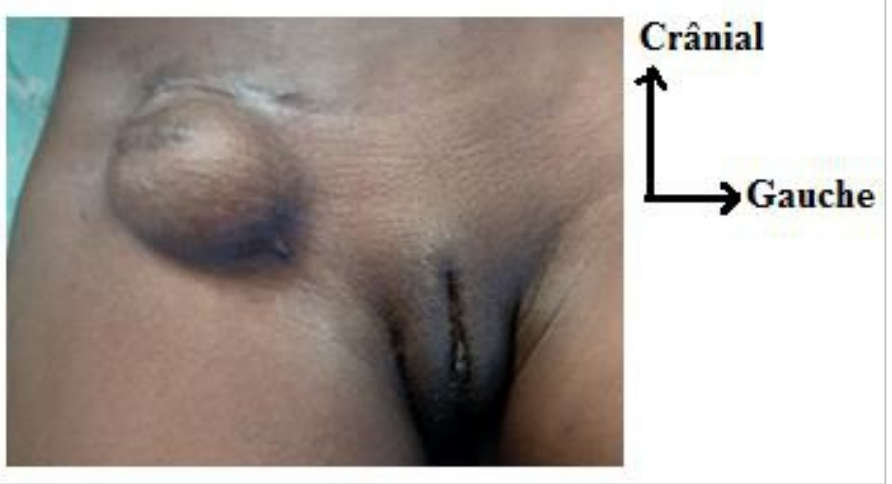

Figure 3. Hernie crurale droite 


\section{Aspects thérapeutiques}

En ce qui concerne le traitement, 13 patients étaient opérés en urgence, et 148 au cours du programme opératoire. Concernant la technique opératoire, tous les patients ont été opérés par voie d'abord ouverte antérieure. Il s'agissait d'un abord transversal dans la plupart des cas (157 cas / 97,52\%). L'abord oblique a été réalisé chez 4 patients adultes qui présentaient de volumineuses hernies inguino-scrotales étranglées. Le traitement du sac herniaire consistait chez l'enfant à la dissection et ligature du canal péritonéovaginal chez le garçon et du canal de Nück chez la fille. Chez l'adulte, il consistait en une dissection et résection ou enfouissement du sac. Pour la réparation pariétale, la technique de Shouldice a été utilisée dans 147 cas $(91,30 \%)$ pour le traitement des hernies inguinales et inguino-scrotales, et la technique de Mac Vay dans 7 cas de hernie crurale et dans 2 cas de hernie inguino-scrotale récidivée. La technique de Bassini a été utilisée dans 6 cas de hernie inguinale, et la technique de Marcy-Little dans un cas de hernie crurale. Deux cas de hernie inguinale récidivée ont été traités par hernioplastie selon Lichtenstein. Des gestes associés ont été faits notamment : la résection épiploïque ( 2 cas), la résection-anastomose du grêle (10 cas dont 8 par voie inguinale et 2 par laparotomie médiane sous-ombilicale), lorsque la hernie était étranglée avec une sphacèle du contenu du sac herniaire; et la circoncision (4 cas) sur demande des parents ou du patient. La Figure 4 est une vue peropératoire d'une anse sphacélée par étranglement herniaire inguino-scrotal.

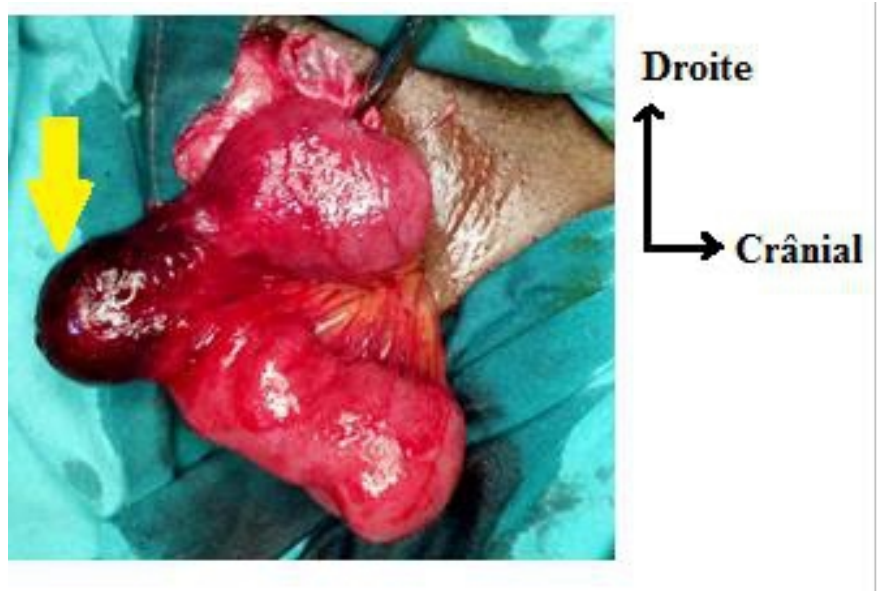

Figure 4. Vue per-opératoire d'une anse sphacélée (flèche) par étranglement herniaire inguino-scrotal

\section{Aspects évolutifs}

Au plan évolutif, les suites opératoires étaient simples dans 157 cas $(97,52 \%)$. Une complication a été retrouvée à court terme dans 4 cas $(2,48 \%)$ 
dont 3 cas $(1,86 \%)$ d'hématome de bourse et un cas $(0,62 \%)$ d'infection du site opératoire. Ces complications avaient bien évolué sous surveillance et traitement médical. La durée moyenne de séjour hospitalier était de 4,53 \pm 1,07 jours (extrêmes : 3 et 9 jours). A moyen et long terme, aucune complication n'a été retrouvée après un recul moyen de 6,15 $\pm 1,69$ mois.

Sur le plan pronostic, la mortalité post-opératoire était nulle. La morbidité post-opératoire était de $2,48 \%$. Le suivi post-opératoire n'a pas noté de particularités cliniques majeures avec un recul moyen de 6,15 $\pm 1,69$ mois (extrêmes : 3 mois et 10 mois).

\section{Discussion}

En Afrique rurale, où, en raison d'un accès aux soins plus difficile, il est estimé que moins d'une hernie sur cinq est opérée (Goutorbe, 2013), la présence des hôpitaux confessionnels constituerait un atout pour les populations. Les hernies de l'aine étaient les plus fréquentes des hernies de la paroi abdominale dans notre étude. Elles touchaient tous les âges avec une prédilection pour le sujet de la troisième à la sixième décennie, et affectaient plus l'homme que la femme. Les couches socio-professionnelles les plus touchées étaient celles exerçant des activités de force. La prédominance masculine et l'atteinte majoritaire des personnes exerçant des activités de force concordent avec d'autres études (Traoré, 2015 ; Mukuku, 2014 ; OloryTogbé, 2010 ; Boukinda, 1993). Si chez l'enfant les hernies de l'aine sont congénitales et liées à la persistance d'un canal péritonéo-vaginal perméable, chez l'adulte, elles sont pour la plupart acquises et sont dues à l'affaiblissement du fascia transversalis au niveau de l'orifice de Fruchaud par des microtraumatismes répétés provoqués par une hyperpression intraabdominale (Wind, 2002; Michel, 2000). Ce qui expliquerait l'atteinte préférentielle des personnes exerçant des activités de force.

La prise en charge des hernies de l'aine a connu des avancées sur le plan chirurgical avec l'avènement des plaques prothétiques et la cœlioscopie. Le traitement des hernies pose aujourd'hui la question de choix de technique parmi plusieurs techniques. Cependant, le choix des procédés thérapeutiques dépend principalement des réalités locales, notamment du plateau technique. Ainsi, en milieu rural voire dans les pays en développement, la cure de hernie de l'aine selon les techniques classiques reste d'actualité (Goutorbe, 2013 ; Traoré, 2015). Chez l'enfant, le traitement repose sur la dissection et la ligature du canal péritonéo-vaginal chez le garçon et du canal de Nück chez la fille (Michel, 2000). Chez l'adulte, le problème se pose au niveau du choix de la technique de réparation pariétale. Cette réparation pariétale peut se faire selon les techniques classiques d'herniorraphie, ou selon les techniques de plasties par plaques (Fingerhut, 2008). L'indisponibilité fréquente des plaques prothétiques en milieu rural fait que les hernioplasties sont rarement 
pratiquées. L'usage des prothèses pour les cures de hernies est peu développé en Afrique (Diop, 2012). La technique de Shouldice est préférée pour le traitement des hernies inguinales en raison d'un faible taux de récidives avec cette technique (Traoré, 2015 ; Shouldice, 2003 ; Simons, 1996).

Les complications postopératoires retrouvées dans notre série ont également été rapportées dans d'autres études (Traoré, 2015 ; Sani, 2007 ; El Alaoui, 1995) avec des proportions supérieures aux nôtres. Il s'agit donc de complications souvent habituelles dans les suites opératoires d'une hernie de l'aine. Même si leur évolution est bonne, des précautions devraient être prises afin de limiter au maximum leur survenue.

\section{Conclusion}

Les hernies de l'aine sont des affections fréquentes en milieu rural où les populations vivent essentiellement des activités de force. Leur diagnostic est clinique et la prise en charge repose sur les techniques chirurgicales classiques. La technique de Shouldice constitue la technique de choix pour le traitement des hernies inguinales en zone rurale où les prothèses sont indisponibles. L'évolution après la prise en charge est le plus souvent favorable.

\section{Conflit d'intérêt : aucun}

Remerciements : Nos remerciements à tout le personnel du service de chirurgie générale, du service de pédiatrie, et du bloc opératoire de l'Hôpital Saint Jean de Dieu d'Afagnan.

\section{References:}

1. Adesunkammi, AR., Badmos, TA., \& Salako, AA. (2007). Groin hernias in patients 50 years of age and above pattern and outcome of management in 250 consecutive patients. West Afr J Med; 19(2) : 142- 7.

2. Boukinda, F., Fagniez, PL., \& Julien, M. (1993). Profil épidémiologique des hernies au Centre Hospitalier de Talangaï à Brazzaville. Etude portant sur 260 malades opérés en 12 mois. Méd Afr Noir; 40(11) : 655 - 61.

3. Diop, B., Sall, I., Ba, PA. et al. (2012). Prise en charge des hernies inguinales géantes : à propos de cinq observations. Méd Santé Trop; $23: 30-34$.

4. El Alaoui, M., Berrada, S., El Mouatacim, K., \& Kadiri, B. (1995). La technique de Shouldice dans le traitement des hernies inguinales à propos de 130 cas. Méd du Maghreb; $53: 6-8$. 
5. Fingerhut, A. \& Pélissier, E. (2008). Traitement chirurgical des hernies inguinales : choix d'un procédé. EMC (Elsevier Masson SAS, Paris), Techniques chirurgicales - Appareil digestif, 40-138.

6. Goutorbe, P., Lacroix, G., Pauleau, G. et al. (2013). Cure de hernie inguinale en environnement difficile : faisabilité et efficience du bloc ilio-inguinal et ilio-hypogastrique. Med Sante Trop; 23 : 202 - 5 .

7. Michel, JL., Lapointe, S., \& De Napoli-Cocci, S. (2000). Hernies de l'enfant. Encycl Méd Chir (Editions Scientifiques et Médicales Elsevier SAS, Paris), Pédiatrie, 4-018-A-10, 7 p.

8. Mukuku, OK., Wakunga, E., Bugeme, M., Kitembo, M., Kabwit, D., \& Wakunga, W. (2014). Hernies inguinales à l'hôpital Jason Sendwe de Lubumbashi en République Démocratique du Congo. A propos de 41 cas. Rev Méd Gd Lacs; 3(1) : $114-25$.

9. Olory-Togbé, JL., Gbessi, DG., Lawani, I., \& Padonou, N. (2010). Hernies pariétales au CNHU HKM de Cotonou. J Afr Chir Digest; 10(2) : $1104-08$.

10. Sani, R., Gbane, M., Hennaux, M., De Groof, D., Mansour, A., \& Ben Ousmane, I. (2007). Prise en charge des hernies inguinales simples : comparaison des aspects économiques et thérapeutiques d'un hôpital de district et l'Hôpital National de Niamey du Niger. Méd Afr Noire; 54 (4) : $215-9$.

11. Shouldice, EB. (2003). The Shouldice repair for groin hernias. Surg Clin North Am; 83(5) : $1163-87$.

12. Simons, MP., Kleijnen, J., van Geldere, D., Hoitsma, HF., \& Obertop, H. (1996). Role of the Shouldice technique in inguinal hernia repair : a systematic review of controlled trials and a meta-analysis. Br J Surg.; 83(6) : $734-8$.

13. Traoré, D., Diarra, L., Coulibaly, B. et al. (2015). Hernies inguinales en Afrique subsaharienne: quelle place pour la technique de Shouldice? Pan Afr Méd J; 22: 50. doi :10.11604/pamj.2015.22.50.6803.

14. Wind, P. \& Chevrel, JP. (2002). Hernies de l'aine de l'adulte. Encycl Méd Chir (Editions Scientifiques et Médicales Elsevier SAS, Paris), Gastro-entérologie, 9-050-A-10, 10p. 


\section{Annexe}

Tableau I. Récapitulatif des paramètres épidémiologiques

\begin{tabular}{|c|c|c|c|}
\hline & 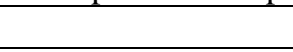 & Effectif & $\%$ \\
\hline \multirow[t]{2}{*}{ Sexe } & Masculin & 146 & 90,68 \\
\hline & Féminin & 15 & 9,32 \\
\hline \multirow[t]{6}{*}{ Tranche d'âge } & {$[1,15[$} & 21 & 13,04 \\
\hline & {$[15,30[$} & 25 & 15,53 \\
\hline & {$[30,45[$} & 38 & 23,60 \\
\hline & {$[45,60[$} & 48 & 29,81 \\
\hline & {$[60,75[$} & 23 & 14,29 \\
\hline & $\geq 75$ & 6 & 3,73 \\
\hline \multirow[t]{10}{*}{ Profession } & Agriculteurs & 27 & 16,77 \\
\hline & Mécaniciens & 22 & 13,66 \\
\hline & Revendeurs & 21 & 13,04 \\
\hline & Menuisiers & 19 & 11,80 \\
\hline & Enseignants & 16 & 9,94 \\
\hline & Maçons & 16 & 9,94 \\
\hline & Pasteurs & 11 & 6,83 \\
\hline & Elèves / Etudiants & 9 & 5,59 \\
\hline & Retraités & 6 & 3,73 \\
\hline & Autres* & 14 & 8,70 \\
\hline \multirow[t]{3}{*}{ Type de hernie } & H. inguinale & 52 & 32,30 \\
\hline & H. inguino-scrotale & 101 & 62,73 \\
\hline & H. crurale & 8 & 4,97 \\
\hline \multirow[t]{3}{*}{ Côté atteint } & Droit & 88 & 54,66 \\
\hline & Gauche & 58 & 36,02 \\
\hline & Bilatéral & 15 & 9,32 \\
\hline
\end{tabular}

Autres* $=$ Nourrissons, enfants non scolarisés, adultes sans emploi. 